\title{
The role of print advertising in clinical trial recruitment: Lessons from a South African site
}

This article was published in the following Dove Press journal:

Open Access Journal of Clinical Trials

21 May 2010

Number of times this article has been viewed

\section{Lesley J Burgess \\ Nicky U Sulzer}

TREAD Research/Cardiology Unit, Department of Internal Medicine, Tygerberg Hospital and Stellenbosch University, Parow, South Africa
Correspondence: Lesley J Burgess TREAD Research/Cardiology Unit, Department of Internal Medicine, Tygerberg Hospital and Stellenbosch University, Parow, South Africa

$\mathrm{Tel}+2721$ 93। 7825

Fax +27 21 9333597

Email lesley@treadresearch.com
Objective: To evaluate the effectiveness of print advertising in our setting in enrolling patients into a study and whether the associated costs are justified.

Methods: This study was carried out by TREAD Research, based within an academic hospital in the Western Cape, South Africa, between January 2006 and June 2007. Newspaper advertising was used as a means of patient recruitment during this period. All advertisements published were summarized according to the total number of advertisements placed, patient response, and the number of patients enrolled compared to the number of patients enrolled from other recruitment methods.

Results: A total of 53 print advertisements were placed over an 18-month period, with a response rate of 1,009 calls. The highest number of responses on average was received in response to the T2DM (type II diabetes mellitus) advertisements (41.2\%). Print advertising contributed more than $75 \%$ of the total number of patients randomized by the site. Apart from one study, print advertising contributed the majority of patients compared with other recruitment methods. The estimated cost of these print advertisements was ZAR 229,482.00 (USD 30,597.60), whereas the estimated income from the patients randomized from these advertisements was ZAR 4,534, 933.00 (USD 604,657.73).

Conclusion: Print advertising, in this setting, was shown to be an expensive but highly effective recruitment method in comparison with other recruitment methods (550\% yield). Its effectiveness is, however, dependent on therapeutic area and patient population.

Keywords: print advertising, recruitment, costs, patient enrollment

\section{Introduction}

Patient recruitment is an essential yet challenging task that is more costly and time consuming than any other aspect of a clinical trial. Pharmaceutical sponsor companies stand to lose up to US\$1.3 million per day that a clinical trial delays a drug's development and registration. ${ }^{1}$ In the United States, the average drug is tested on 4,000 study subjects during 37 clinical trials before receiving Food and Drug Administration (FDA) approval. ${ }^{1}$ Subject recruitment often proves to be the rate-limiting step in many trials and is recognized as the leading bottleneck in the new drug development pipeline..$^{1-3}$ It has been reported that up to $80 \%$ of clinical trials do not meet their enrollment quotas on time, ${ }^{4}$ and the majority of clinical trials run late by an average of 6 weeks. ${ }^{5}$ Added to this is the fierce competition between pharmaceutical companies to produce the necessary scientific data within the quickest possible timeframe. It is thus understandable that increasingly more pressure is put on research sites to produce the required number of patients within the scheduled timeframe. 
Pharmaceutical companies have embraced globalization as a core component of their business models and the number of countries serving as trial sites outside the United States has more than doubled in the past ten years. ${ }^{6}$ Globalization is motivated primarily by substantial cost savings, a large pool of potential participants who can be rapidly recruited in shortened timelines and as a way to overcome regulatory barriers in these developing countries which offer potentially lucrative markets. ${ }^{6,7}$

To meet this demand, research sites in developing countries specifically need to develop an effective and practical recruitment plan, taking the protocol requirements and local patient population into account. In some instances, the site's existing database is insufficient and specific recruitment strategies need to be implemented. Some of these include hanging posters in waiting areas, handing out brochures, writing letters to referring doctors, visiting community clinics and placing print advertisements in the local press and more recently, the use of the internet in attracting potential patients' interest. Each of these methods has a potential role to play in the overall recruitment strategy although some methods may prove more effective than others.

\section{Objective}

The primary objective of this study was to evaluate the effectiveness of print advertising in enrolling patients at our research site compared with other recruitment methods. The secondary objective was to determine whether the costs associated with this form of advertising are justified in terms of patient recruitment.

\section{Methods}

This study was carried out by TREAD Research, a Site Management Organization (SMO) based within an academic hospital in the Western Cape, South Africa, between January 2006 and June 2007. Newspaper advertising was used as a means of patient recruitment for five different therapeutic areas during this period. These included (i) primary prevention of ischemic heart disease (IHD) and stroke, (ii) hypercholesterolemia in adults, (iii) hypercholesterolemia in children, (iv) type II diabetes mellitus (T2DM), and (v) hypertension. All advertisements were approved by the relevant sponsor companies and the University of Stellenbosch's Committee for Clinical Trials.

All telephonic responses to these advertisements were answered by a dedicated staff member who completed a specific prescreen form with potential trial participants. Each study had a dedicated prescreen form which was designed prior to the placement of any advertisement. This form included questions regarding patient contact details, date of birth, known medical history and concomitant medication, as well as any protocol-specific requirements. The outline of the study was explained to potentially eligible patients and they were asked whether they would like to attend a screening visit. During the screening visit, patients are asked to read the study's informed consent form, an investigator conducts the informed consent process with them and once they have decided to participate, various protocol-mandated procedures are conducted.

All advertisements published were summarized according to the total number of advertisements placed, patient response (number of phone calls received) to each advertisement, and the number of patients that were enrolled into the respective studies. Furthermore, the number of patients enrolled into these therapeutic areas from print advertising was compared to the number of patients enrolled from various other recruitment methods, namely patient referrals, our site's database, outpatient clinic referrals, word of mouth and day hospitals. Data was compiled by a data capturer and descriptive statistics were used to analyze the data.

The cost of placing these advertisements was recorded and the income generated from patients randomized onto the studies from these advertisements was calculated. This was done by multiplying the per-patient fee as per individual study budget by the number of patients randomized onto the trial. The percentage yield was then calculated using these figures.

\section{Results}

Table 1 presents a summary of the print advertisements placed over this specific time period and the response received to these advertisements per therapeutic area. A total of 53 print advertisements was placed over an 18-month period, with a total response rate of 1,009 calls. The highest number of responses on average was received in response to the T2DM advertisements $(41.2 \%)$, while the response to the hypercholesterolemia study in children received an average of only two calls per advertisement. In addition, there were 20 advertisements placed for the single primary prevention of heart disease study compared with 12 advertisements for six T2DM studies.

The contribution of these print advertisements to patient randomization is presented in Table 2. The site met all of the trials' recruitment targets except for the hypercholesterolemia study in children where just four of the required 10 patients were randomized. In this case, no patients were randomized 
Table I Summary of print advertisements placed and their response (number of phone calls)

\begin{tabular}{lllll}
\hline Therapeutic area & $\begin{array}{l}\text { Number of } \\
\text { studies }\end{array}$ & $\begin{array}{l}\text { Number of } \\
\text { advertisements } \\
\text { placed }\end{array}$ & $\begin{array}{l}\text { Response } \\
\text { (\# of calls) }\end{array}$ & $\begin{array}{l}\text { Average response } \\
\text { per advertisement } \\
\text { (\# of calls) }\end{array}$ \\
\hline Primary prevention of IHD and stroke & 1 & 20 & 338 & 16.9 \\
Hypercholesterolemia in adults & 2 & 9 & 124 & 13.7 \\
Hypercholesterolemia in children & 1 & 2 & 4 & 2 \\
Type II diabetes mellitus & 6 & 12 & 494 & 41.2 \\
Hypertension & 2 & 10 & 49 & 4.9 \\
Total & $\mathbf{1 2}$ & $\mathbf{5 3}$ & $\mathbf{1 0 0 9}$ & $\mathbf{1 9 . 0 4}$ \\
\hline
\end{tabular}

Abbreviation: IHD, ischemic heart disease.

from print advertising responses. In all other studies, print advertising contributed more than $75 \%$ of the total number of patients randomized by the site, with more than $110 \%$ of the required number of patients for each study coming from print advertisements.

Figure 1 presents the relative contribution of print advertising to total randomization numbers, in comparison with other recruitment methods. Apart from the hypercholesterolemia in children study, print advertising contributed the majority of patients compared with other recruitment methods, including patient referrals, our site's database, outpatient clinical referrals, word of mouth and patients sourced from local day hospitals.

The approximate cost of these print advertisements was ZAR 229,482.00 (USD 30,597.60, assuming 1 USD = ZAR 7.50), approximately ZAR 4,500.00 (USD 600.00) per advertisement whereas the estimated income from the patients randomized from these advertisements was ZAR 4,534,933.00 (USD 604,657.73). The percentage yield was 550\%.

\section{Discussion}

The results of this study show that, in this setting, print advertising is extremely effective in enrolling patients into particular trials compared with other recruitment methods. In addition, the cost associated with print advertising was found to be justified in terms of the revenue generated from this recruitment method (550\% yield).

Patient recruitment and retention in clinical trials is widely recognised as the leading bottleneck in the new drug development pipeline, and it is likely to remain an area of heightened concern. ${ }^{3}$ Clinical drug testing and regulatory approval processes are more complex and expensive than ever, with regulatory agencies requiring larger research subject populations and longer periods of treatment before approving new drugs. ${ }^{1,8}$ The number of clinical trials per new drug application has doubled in the decade 1985-1995. ${ }^{9}$ It is estimated that patient recruitment consumes $27 \%$ of the annual cost of development worldwide but only one in 20 recruited patients provides results that can be included in a regulatory dossier. ${ }^{8}$ Each patient enrolled into a clinical trial represents a significant amount of time, effort and other resources. ${ }^{10}$ Patient drop-outs and lost-to-follow-up after recruitment negatively affect study duration, study cost and the generalising of study results which may result in a delay in the drug's regulatory approval. ${ }^{10}$ All of this in turn means that research sites are required to enroll more patients into studies and that meeting enrollment deadlines and maintaining high retention rates becomes essential to a site's survival in the clinical trial industry.

Table 2 Summary of print advertisements' contribution to patient randomization totals

\begin{tabular}{|c|c|c|c|c|c|}
\hline Therapeutic area & $\begin{array}{l}\text { Sponsor patient } \\
\text { target per site }\end{array}$ & $\begin{array}{l}\text { Number of } \\
\text { patients } \\
\text { randomized } \\
\text { at site }\end{array}$ & $\begin{array}{l}\text { Patients } \\
\text { randomized from } \\
\text { advertisements }\end{array}$ & $\begin{array}{l}\text { \% sponsor target } \\
\text { randomized from } \\
\text { advertisements }\end{array}$ & $\begin{array}{l}\% \text { total patients } \\
\text { at site from } \\
\text { advertisements }\end{array}$ \\
\hline $\begin{array}{l}\text { Primary prevention of IHD and } \\
\text { stroke }\end{array}$ & 50 & 78 & 60 & 120 & 76.9 \\
\hline Hypercholesterolemia in adults & 20 & 39 & 33 & 165 & 84.6 \\
\hline Hypercholesterolemia in children & 10 & 4 & 0 & 0 & 0 \\
\hline Diabetes mellitus type II & $\begin{array}{l}\text { Unlimited } \\
\text { (competitive) }\end{array}$ & 54 & 49 & $\begin{array}{l}\text { Unlimited } \\
\text { (competitive) }\end{array}$ & 90.7 \\
\hline Hypertension & 31 & 40 & 36 & 116 & 90 \\
\hline
\end{tabular}

Abbrevation: IHD, ischemic heart disease. 


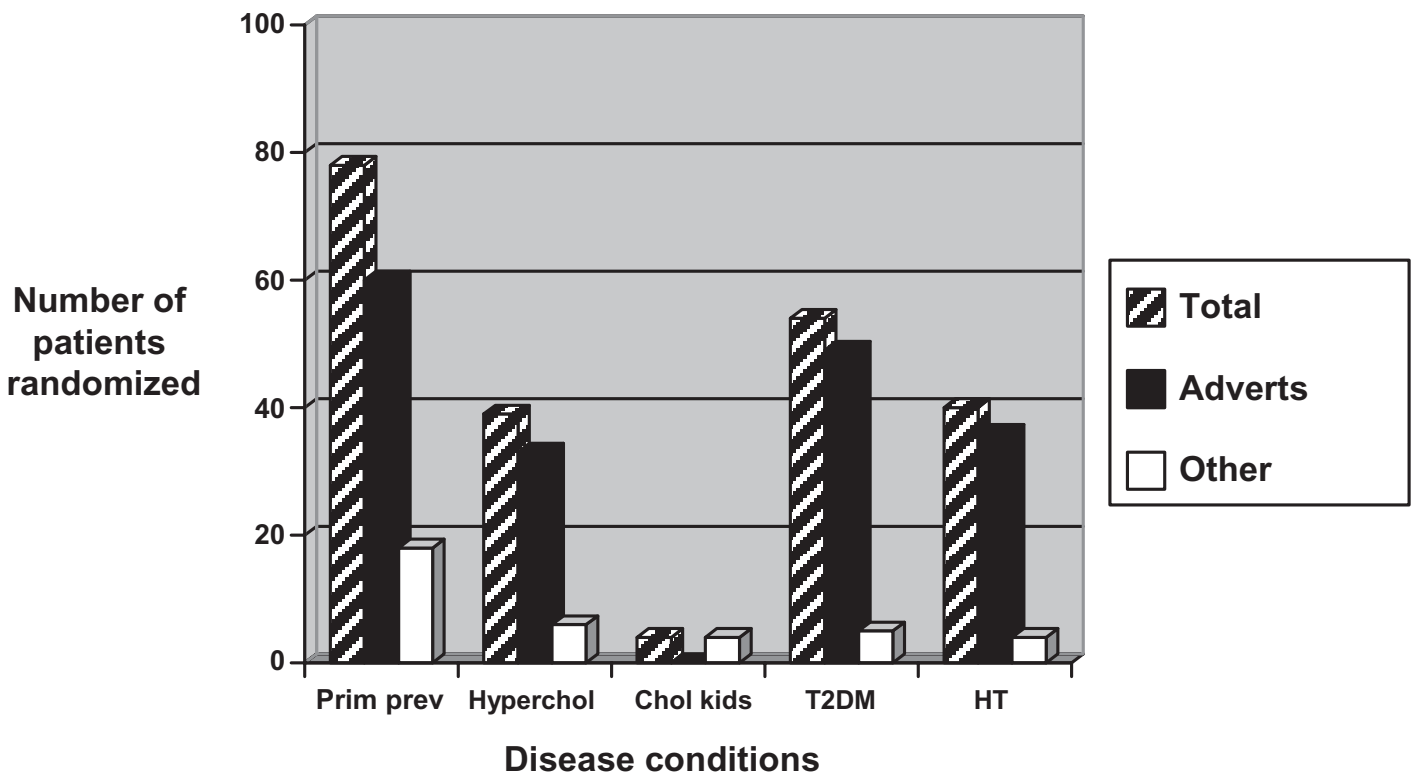

Figure I Number of patients randomized in total and the relative contribution of print advertising versus other recruitment methods. Abbreviations: Chol, cholesterol; HT, hypertension; T2DM, type 2 diabetes mellitus.

In this setting, print advertising was shown to be more effective than the other available recruitment methods in enrolling patients onto particular studies. This finding is supported by recent studies which also report on the effectiveness of print advertising in enrolling patients. ${ }^{11,12}$

The results of the current study do show, however, that the effectiveness of print advertising is not the same for all therapeutic areas. Print advertisements failed to enroll a single patient onto the hypercholesterolemia in children study. In the case of a highly competitive therapeutic area, namely primary prevention of heart disease, 20 advertisements were necessary for one study. These findings have been reported previously where it was found that median time for enrollment varies with therapeutic field. ${ }^{9}$ It has also been suggested that specialist niche areas may require a more refined recruitment strategy. ${ }^{2}$

The success of any given recruitment method may vary based on the target population, ${ }^{12}$ and the method of recruitment selected should take the demographics and cultural nuances of a particular patient population into account. ${ }^{2,13,14}$ In this particular instance, print advertising was shown to be highly effective in enrolling patients onto certain trials. However, the effectiveness of this recruitment method may well differ in other patient populations. This emphasizes the idea that when it comes to recruitment, "one size does not fit all". ${ }^{15}$ An article outlining subject recruitment issues in India highlights what the authors refer to as "India-specific factors." 16 This emphasizes the need for research sites to know their patient population and what form of recruitment will work best in their situation. Additionally, recruitment methods that work for a private practitioner will be different to those that work for an academic medical centre and will differ to those that work for a dedicated trial site. ${ }^{15}$

A prime example of this is Internet-based advertising. Internet-based recruitment methods have been used by this site, albeit unsuccessfully. A pilot project using this recruitment method produced only one patient. In addition, in many developing countries, poor telecommunication infrastructure prevents the successful use of this strategy. Internet-based recruitment has, however, been shown to be highly effective in Europe and the United States. ${ }^{1}$ In order for any recruitment campaign to be successful, it is imperative therefore to know which medium will have the most influence on the target audience, taking cultural influence, local infrastructure and the specific setting into account. ${ }^{14}$

Despite the success rate of print advertising in this setting, the findings of this study emphasize that print advertising as a recruitment method is a labor intensive process. This is indicated, for example, by the fact that of the 338 responses to the primary prevention of IHD and stroke advertisements, only 60 patients were finally randomized. Despite the fact that many respondents are ineligible for screening due to medical history, concomitant medication or protocol-specific criteria, many respondents have been known to misread or misinterpret the advertisement and cannot be prescreened by telephone.

It has been reported that advertising costs are the single greatest expense for many subject enrollment programs. ${ }^{9}$ 
Although that statement probably refers to the more expensive television advertising, the costs of print advertising locally are also high (an average of USD 600.00 per advertisement). It must also be noted that the cost of the advertisement is not the only cost involved in this method of recruitment. One also needs to factor in the time involved for a dedicated person to receive and respond to these phone calls, as well as the cost of the actual calls themselves. These logistical issues will also directly affect the effectiveness of any given recruitment method. For example, a print advertisement may have a large response from the public but if there is insufficient site staff to screen potential patients or if the staff do not respond promptly, potential patients may be lost. In such an instance, the recruitment figures would not be an accurate reflection of the potential effectiveness of this recruitment method. ${ }^{15}$ In this setting with a solid infrastructure, the cost of print advertising was found to be justified and the income generated by the randomized patients by far outweighed the expenses incurred.

Print advertising is, however, just one of the recruitment methods available to research sites. Others include patient booklets, brochures, community outreach programmes, posters for waiting areas, letters to referring physicians and more recently Internet-based advertising. ${ }^{13,17}$ The effectiveness of any given method must be tried and tested to determine which method will work best in a particular situation with a particular population.

\section{Conclusion}

Print advertising, in this setting, was shown to be an expensive but highly effective recruitment method in comparison with other recruitment methods. Its effectiveness is, however, dependent on therapeutic area and patient population. As the demand for increasing patient numbers grows and trial enrollment deadlines become more stringent, research sites need to make use of market research tools to formulate proactive recruitment strategies that take their particular patient population into account. In so doing, they will hopefully be able to meet one of clinical trials' greatest challenges by recruiting the required number of patients within the stipulated timeframe.

\section{Acknowledgments}

The authors report no conflicts of interest in this work. The authors would like to acknowledge M Ambrose's assistance with data collection.

\section{References}

1. Smit-Marshall P. Recruitment Strategies: A European Perspective Applied Clinical Trials. 2006 Feb 1. Accessed on June 20, 2008. Available from: http://www.actmagazine.com/appliedclinicaltrials/article/.

2. Sergeant E. Methods of clinical trial recruitment that have and will impact the pharmaceutical industry. Drug Development. 2007: $38-40$.

3. Research and Markets' Resources Division. Challenges in patient recruitment for clinical trials: overcoming the Bottleneck. 2005 Dec. Accessed Jan 8, 2010. Available from: http://www.researchandmarkets.com/.

4. Bowman K. Choice of control group in clinical trials and patient recruitment rates. International Biopharmaceutical Association Publication. 2007 June 12. Accessed June 20, 2008. Available from: http:// www.ibpassociention.org/.

5. Getz KA. Is investigative site feasibility feasible? Applied Clinical Trials. 2008 July 1. Accessed on Aug 13, 2008. Available from: http://www. actmagazine.com/appliedclinicaltrials/article/.

6. Glickman SW, et al. Ethical and scientific implications of the globalization of clinical research. $N$ Engl J Med. 2009;360(8):816-823.

7. Petryna A. When Experiments Travel: Clinical trials and the global search for human subjects. 1st ed. Princeton, NJ: Princeton University Press; 2009.

8. Drayton S, Fraser HE. Five steps to faster recruitment. GCP Journal. 2005;Feb:17-20

9. Bonacossa P, Kermani F. New Ways to Recruit Trial Subjects. Applied Clinical Trials. $2003 \mathrm{Feb}$ 1. Accessed on June 20, 2008. Available from: http://www.actmagazine.com/appliedclinicaltrials/article/.

10. Betz D, Betz D, Leahy D. Burden of patient dropouts and patients lostto-follow-up on clinical studies. Accessed on Feb 17, 2010. Available from: http://www.lost-to-follow-up.com/.

11. McDermott MM, et al. Recruiting participants with peripheral arterial disease for clinical trials: experience from the Study to Improve Leg Circulation (SILC). J Vasc Surg. 2009;49(3):653-659.

12. Cambron JA, et al. Recruitment methods and costs for a randomized, placebo-controlled trial of chiropractic care for lumbar spinal stenosis: a single-site pilot study. J Manipulative Physiol Ther. 2010;33(1): $56-61$.

13. Jones J. Access all areas: Global patient recruitment. GCP Journal. 2006; June:35-38.

14. Beasley D. Reaching an African-American audience. GCP Journal. 2006;Feb:18-21.

15. Eriksson LO, Harper BD. Successful subject recruitment: one size does not fit all. Applied Clinical Trials Online. 2002 Nov 1. Accessed Mar 1, 2010. Available from: http://www.appliedclinicaltrialsonline.com/.

16. Sahoo U, Sanghavi V, Kermani F. Subject recruitment: an indian perspective. Applied Clinical Trials Online. 2006 Feb 1. Accessed Mar 1, 2010 Available from: http://www.appliedclinicaltrialsonline.com/.

17. Wolf L. "On message" for patient recruitment. GCP Journal. 2006; April:22-25.

Open Access Journal of Clinical Trials

\section{Publish your work in this journal}

The Open Access Journal of Clinical Trials is an international, peerreviewed, open access journal publishing original research, reports, editorials, reviews and commentaries on all aspects of clinical trial design, management, legal, ethical and regulatory issues, case record form design, data collection, quality assurance and data auditing

methodologies. The manuscript management system is completely online and includes a very quick and fair peer-review system, which is all easy to use. Visit http://www.dovepress.com/testimonials.php to read real quotes from published authors. 